Topics

\title{
Current Status on Preparation of Fuel Droplet Clouds Combustion Experiment "Group Combustion" Onboard the KIBO
}

\author{
By Masao KIKUChI $^{1}$, Yuuji KAN $^{1)}$, Aya TAZAKI ${ }^{1)}$, Shin YAMAmoto $^{2)}$, Masaki NOKURA ${ }^{2)}$, Naoshi HANAFUSA ${ }^{2)}$, \\ Yasuyuki HISASHI ${ }^{2)}$, Osamu MORIUE ${ }^{3)}$, Hiroshi NOMURA ${ }^{4)}$ and Masato MIKAMI ${ }^{5)}$ \\ ${ }^{1)}$ ISS Science Project Office, JAXA/ISAS, Tsukuba, Japan \\ ${ }^{2)}$ IHI Scube Co., Ltd., Tachikawa, Japan \\ ${ }^{3)}$ Graduate School of Engineering, Kyushu University, Fukuoka, Japan \\ ${ }^{4)}$ College of Industrial Technology, Nihon University, Narashino, Japan \\ ${ }^{5)}$ Graduate School of Science and Engineering, Yamaguchi University, Ube, Japan
}

(Received June 20th, 2013)

\begin{abstract}
The "Group Combustion" experiment is planned as the first combustion experiment in the KIBO on the ISS. Flame spread characteristics of n-decane droplet clouds and linear array will be investigated in detail. The experiment will employ the Multi-purpose Small Payload Rack (MSPR), the Chamber for Combustion Experiment (CCE), and the Group Combustion Experiment Module (GCEM) as experimental apparatus. At present, development of the GCEM has been on-going in parallel to detail planning of the experiment operation onboard the KIBO. In this paper, current status on preparation of the experiment will be introduced.
\end{abstract}

Key Words: Combustion, Fuel Droplets, Microgravity, ISS, KIBO

\section{Introduction}

Flame propagation among fuel spray at the base of spray flame and subsequent excitation of the group combustion of the whole spray are necessary for stable and complete combustion of continuous burning of liquid fuel such as aero engines or gas turbines. For controlled high efficient combustion, numerical simulation of spray flame, based on the precise understanding of flame spread mechanism among fuel droplets, is important.

In order to clarify the flame spread mechanism among fuel droplets, flame spread of linear droplet array in microgravity environment has been investigated ${ }^{1-9)}$. The merits of microgravity experiments are exclusion of natural convection and magnification of representative scales of the phenomena. In addition to the investigation on linear droplet array, investigation on flame spread of 2 dimensional droplet clouds were performed by Mikami et al., employing short duration microgravity experiments. Figure 1 shows flame spread images of $\mathrm{n}$-decane droplet clouds in microgravity. A percolation model to describe group combustion excitation mechanism of randomly-distributed droplet clouds, which is based on flame spread limit between droplets, was established by them ${ }^{10,11)}$.

"Elucidation of flame spread and group combustion excitation mechanism of randomly-distributed droplet clouds (Group Combustion)", is the investigation to be performed in the KIBO on the ISS (International Space Station). "Group Combustion" is based on the above mentioned ground research results, and would be the first combustion experiment to be executed onboard the KIBO. The experiment was selected as a flight candidate investigation by KIBO research
AO (Announcement of Opportunity) in 2008.

In this paper, overview of the "Group Combustion" experiment and current status on preparation of the experiment will be introduced.

\section{Overview of the Experiment}

The purpose of the "Group Combustion" experiment are to verify the flame spread hypotheses, which are based on the percolation theory and the findings from short-duration microgravity experiments, using large-scale droplet clouds and to develop a percolation model which will describe the group combustion excitation through the flame spread. It also verifies the hypothesis of characteristic time-scale divergence at the critical point based on the percolation theory. The experiment consists of 3 sub-experiments to verify different hypothesis. Overview of each sub-experiment is as follows.
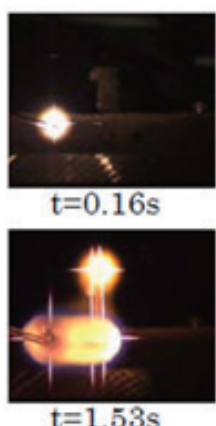

$\mathrm{t}=1.53 \mathrm{~s}$

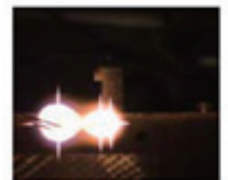

$\mathrm{t}=0.60 \mathrm{~s}$

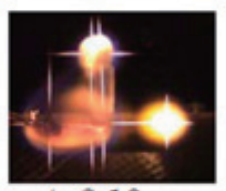

$\mathrm{t}=2.10 \mathrm{~s}$

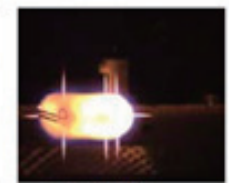

$\mathrm{t}=1.17 \mathrm{~s}$

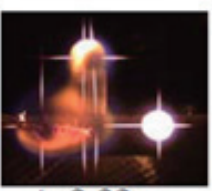

$\mathrm{t}=2.33 \mathrm{~s}$
Fig. 1. Flame spread images of n-decane droplet clouds in microgravity. 
Sub-experiment 1: Clarification of droplet interaction effects on flame spread limit.

Sub-experiment 2: Clarification of the effects of droplet motion on flame spread limit.

Sub-experiment 3: Clarification of the effects of radiative heat loss on flame spread limit.

In the sub-experiment 1 , similar flame spread experiments of droplet clouds as shown in Fig. 1 would be performed systematically, with changing droplet arrangements. Also, a principle of flame spread is verified by using randomly-distributed large scale droplet clouds which contains more than 150 droplets at maximum. In the sub-experiment 2 , interaction between spreading flame and droplet motion are investigated by using linear droplet array which contains movable droplets in the array direction as shown in Fig. $2^{12)}$. In the sub-experiment 3, flame spread from droplet cluster to the other cluster is observed with changing the size of the clusters. Since the radiative heat loss from the flame strongly depends on the flame size, the effect of radiative heat loss on flame spread process will be clarified through such experiments. For all experiments of the Group Combustion, n-decane $\left(\mathrm{C}_{10} \mathrm{H}_{22}\right)$ is employed as fuel.

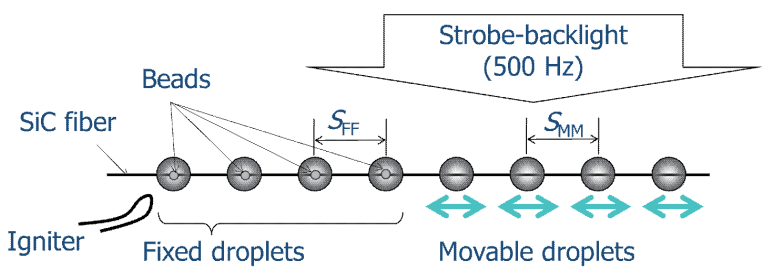

Fig. 2. Schematic of the linear fuel droplet array which contains movable droplets in the array direction.

\section{Experimental System}

\subsection{System overview}

The experiment will employ the Multi-purpose Small Payload Rack (MSPR) and the Chamber for Combustion Experiment (CCE) as parent facilities in the KIBO. The CCE was developed as a component of the MSPR, to accommodate experiment dedicated combustion module inside it. Containment of toxic materials, gas, and fire are major functions of the CCE. Therefore, the CCE is sealed by double O-rings. Also, it provides accommodated experimental module with various interfaces such as gas supply/vent, electrical power supply, communication with ground etc.. The CCE was transported to the ISS by the HTV-2 in 2011, and on-orbit function check has been on-going.

For "Group Combustion", the Group Combustion Experiment Module (GCEM) is employed as a dedicated experiment module. During on-orbit experiment, the GCEM is installed inside the CCE. Then, the CCE is installed at the Work Volume (WV) of the MSPR. Also, gas bottle A assy is installed in the Small Experimental Area (SEA) of the MSPR. The gas bottle A assy consists of a gas bottle, QD, and fitting. The gas bottle itself is a Gas Bottle Unit (GBU), which was originally developed as a JEM system component.

Figure 3 shows schematics of assembly procedures of the relevant experimental system.

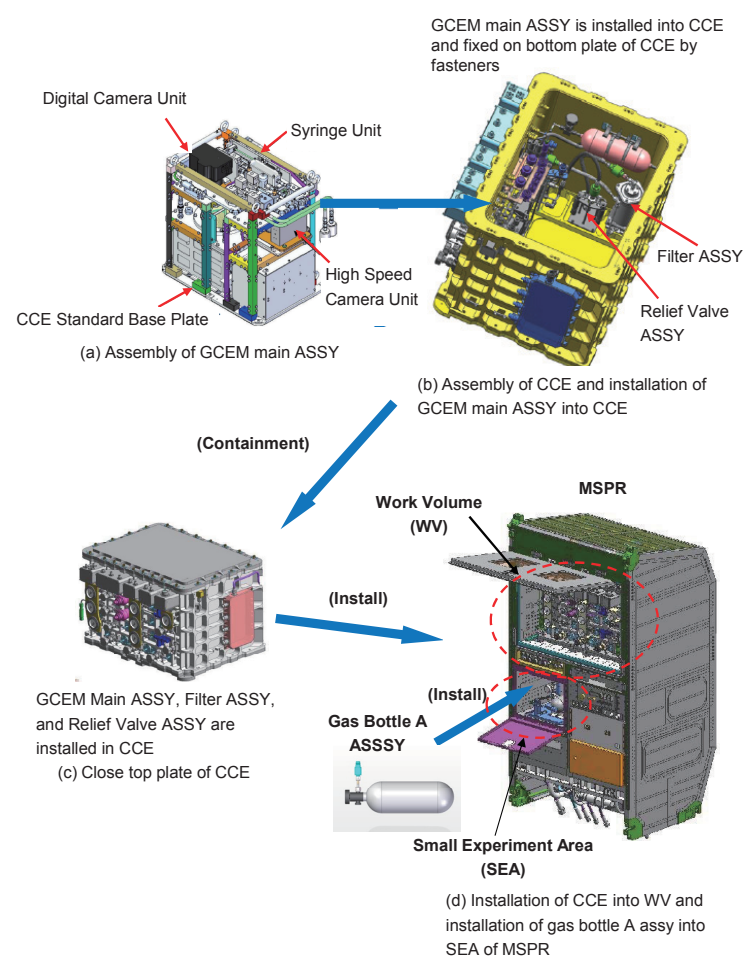

Fig. 3. Schematics of assembly procedures of experimental system.

\subsection{Experiment module}

The GCEM consists of the following sub-systems.

1) Fuel Supply Unit

2) Small Combustion Vessel Assy

3) Observation Unit

4) Gas Inlet/Outlet Line

5) Power Supply and Control Unit

6) Frame Assy

7) Gas Bottle A Assy

8) Filter Assy

Sub-systems 1) - 6) are integrated as the GCEM main assy. Figure 4 shows a schematic of the GCEM main assy. Also, pictures of the engineering model (EM) of the GCEM are shown in Figure 5.

\subsubsection{Fuel supply unit}

Figure 6 shows schematics of fuel supply unit. Fuel supply unit consists of syringe unit, fuel supply mechanism, and fuel supply line. syringe unit consists of an efficient gas tight syringe (COTS), QD, and protection case. The syringe stores $9.5 \mathrm{ml}$ liquid n-decane. It is sealed by double sealed QD (QD female and QD cap). Moreover, the syringe is covered with PTFE tube to prevent the scattering of the glass. It is possible to exchange the syringe unit since it is ORU (Orbital Replacement Unit). Syringe unit is installed onto the fuel supply mechanism.

Fuel supply mechanism consists of ball screw, slide rail, and stepper motor. Plunger of the syringe is pushed by fuel supply mechanism to supply fuel for droplet generation. 


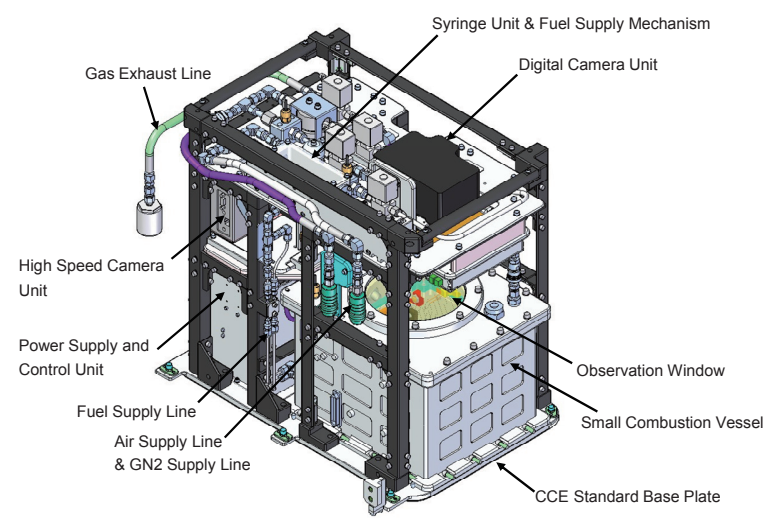

Fig. 4. Schematic of the GCEM main assy.

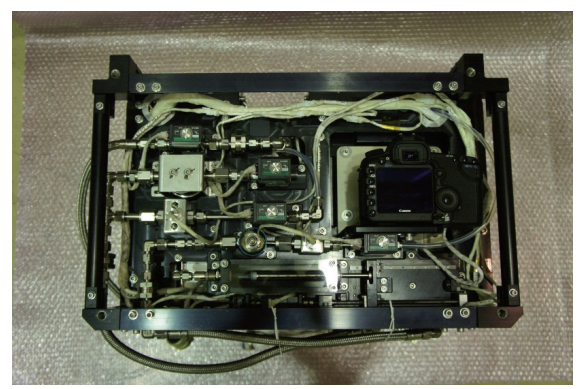

(a) Top view

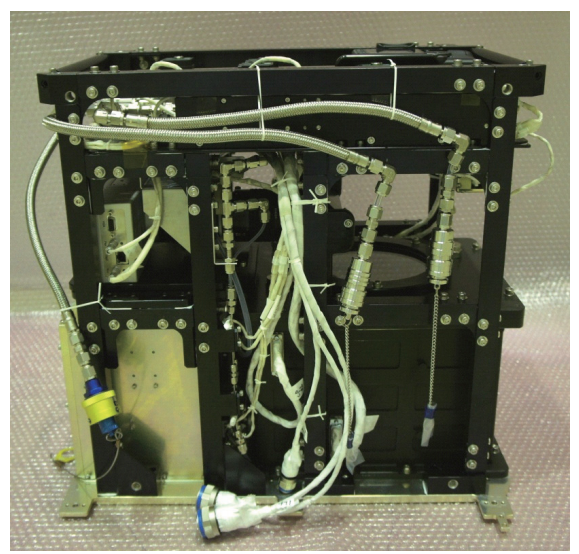

(b) Front view

Fig. 5. Photo of the engineering model (EM) of the GCEM.

Also, fuel supply mechanism is equipped with an encoder to detect the position of plunger.

Fuel supply line consists of QD (Male), PFA tube (plumbing), mesh filter, and isolation valves. During setup phase of the GCEM, QD (Female) of the syringe is connected to QD (Male) of fuel supply line by crew. Mesh filter is used to remove contamination of fuel line. Fuel supply line is divided to two lines for redundancy. Isolation valves are installed to prevent excessive fuel supply.

Fuel supply lines (fuel tubes) are connected into fuel discharge unit which is mounted on traverse mechanism inside small combustion vessel.
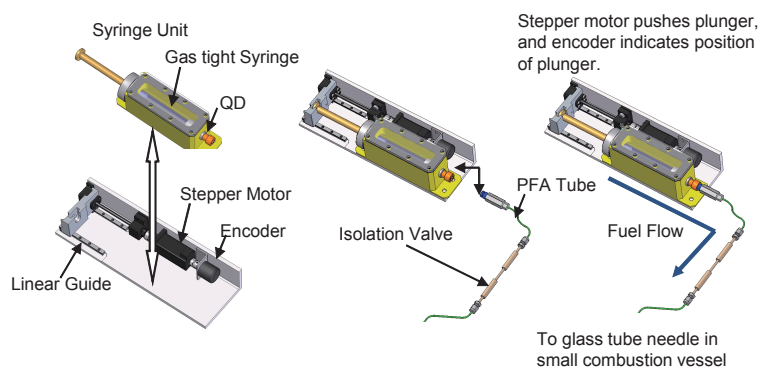

Fig. 6. Schematics of fuel supply unit.

\subsubsection{Small combustion vessel assy}

Small combustion vessel assy consists of main body of small combustion vessel and components which are integrated into the main body. Small combustion vessel is a single sealed vessel. Combustion experiments are actually performed inside the vessel. Inner dimension of the vessel is $270 \times 240 \times 200$ $\mathrm{mm}$. Net inner volume of the vessel is approximately 12.11 . Combustion phenomena inside the vessel is observed through a top window of the vessel, which is made of sapphire glass. MDP (Maximum Design Pressure) of the vessel is $0.2 \mathrm{MPa}$ abs, which is limited by MDP of CCE gas supply line. Two relief valves are installed into the vessel, to release unexpected pressure increase inside the vessel. In case high pressure gas is released inside the CCE through the relief valves, it is possible to clean up inner volume of the CCE by prescribed cleaning procedures. Small combustion vessel assy is ORU, and never opened on orbit.

Components integrated into the main body of the vessel are as follows.
1) Traverse Mechanism
2)Fuel Discharge Unit (2 ea.)
3)Droplet Support Unit
4)Ignition Unit (4 ea.)
5) Stirrer Unit
6) Soot Removal Unit
7) Relief Valves (2 ea., 0.185 MPa abs)
8) Pressure Sensor (2 ea.)
9) Temperature Sensor (2 ea.)

Figure 7 shows a schematic of the traverse mechanism. Traverse mechanism consists of 3 axis stage including stepper motors, ball screws, limit switches, LEDs, and fuel discharge unit. Fuel discharge unit for droplet generation is fixed on stage-z of the traverse mechanism. It is possible for the fuel discharge unit to move 3-dimensionally by the motion of 3 axis stages. Each stage is moved by stepper motor, and equipped with limit switches to detect initial and end positions.

Glass tube needle of the fuel discharge unit is connected to fuel tube by epoxy resin bond. n-Decane as liquid fuel is supplied through fuel tube by the motion of the plunger of the syringe unit. Fuel droplet generated at the tip of the glass tube easily sticks to the $\mathrm{SiC}$ fiber by up and down motion of stage-z. For redundancy, 2 sets of fuel discharge unit are integrated in the vessel. 
LED backlights are fixed on stage-x, and used as follows:

-Measurement of droplet diameter

-Observation of droplets on $\mathrm{SiC}$ fiber

-Observation of combustion phenomena

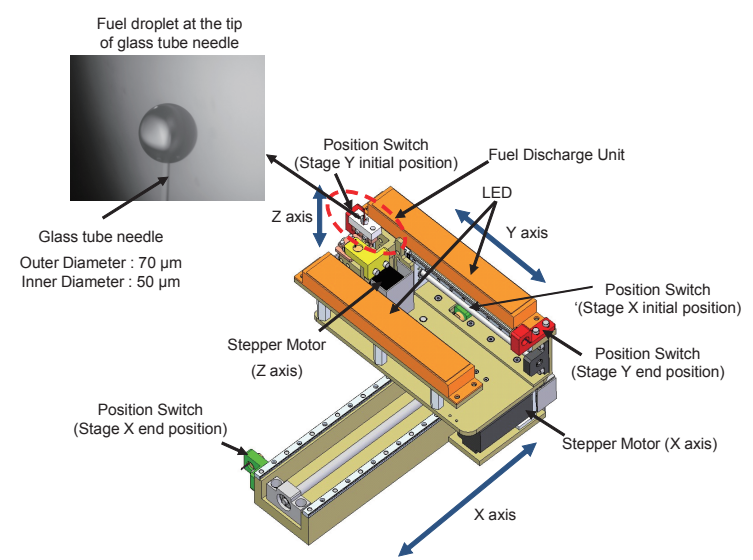

Fig. 7. Schematic of the traverse mechanism and fuel discharge unit.

Figure 8 shows the schematic of the droplet support unit and ignition unit. Two kinds of SiC fibers are integrated in the frame of the droplet support unit. One is $30 \times 30$ lattice of $\mathrm{SiC}$ fibers with 14 micron diameter. Another is a single SiC fiber with 78 micron diameter. Fiber lattice is employed for sub-experiment 1 and 3 for flame spread among fuel droplet clouds or clusters. On the other hand, single fiber is employed for sub-experiment 2 for flame spread along movable linear droplet array. For sub-experiment 1 and 3, fuel droplets are supported on the crossing points of latticed SiC fibers. For sub-experiment 2 , movable droplets are just supported on the fiber, though first 4 droplets as fixed droplets are supported on the ceramic beads on the fiber.

Also, 4 ignition units are integrated in the frame of the droplet support unit. 2 units are for sub-experiment 1 , and the other 2 units are for sub-experiment 2 and 3, respectively. In addition to 1 main unit, another unit is prepared as redundancy for sub-experiment 1 , since test matrix of the sub-experiment 1 is larger than the other sub-experiments. Ignition unit is made of SUS tubes, $\mathrm{Fe}-\mathrm{Cr}$ wire, and PEEK insulator. Resistance of the ignition unit is approximately $1 \mathrm{ohm}$, and the $\mathrm{Fe}-\mathrm{Cr}$ wire works as hot igniter by electric current with $6 \mathrm{~A}$.

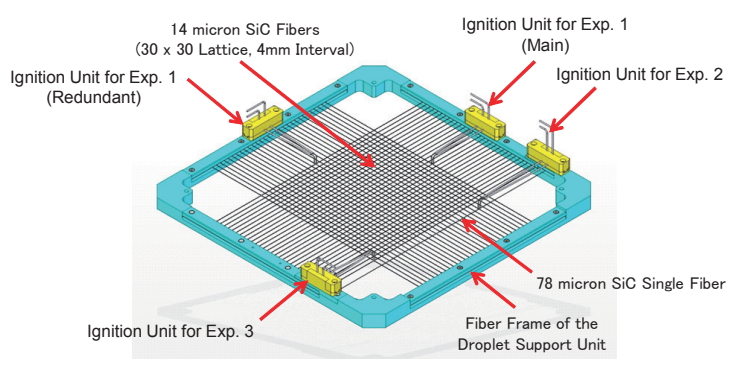

Fig. 8. Schematic of the droplet support unit and ignition unit.

In addition to these components, stirrer unit and soot removal unit are integrated in the small combustion vessel assy. The stirrer unit is very small fan. Functions of the stirrer unit are to circulate gas inside the vessel for mixing atmosphere and to accelerate evaporation of the fuel droplets. Soot removal unit consists of spray nozzle and plumbing. From 2 nozzles, nitrogen gas supplied from the ISS/KIBO through the MSPR/CCE is injected to the SiC fibers to remove soot after combustion experiments as needed.

\subsubsection{Observation unit}

Observation unit of the GCEM includes the digital camera unit and high speed camera unit. The digital camera unit consists of a digital camera (COTS), a lens (COTS), and its manifolds. The camera records video images of droplet generation and combustion phenomena with 30 frames/s as well as still images of the backlit droplets.

The high speed camera unit consists of a high speed video camera (COTS), a lens, and manifolds. The camera is employed for the sub-experiment 2. Spreading flame along the droplet array and backlit droplets are recorded alternately by blinking of the LED with $500 \mathrm{~Hz}$.

\subsubsection{Gas inlet/outlet line}

Gas inlet/outlet line consists of air supply line, GN2 supply line, and gas exhaust line. High pressure air inside the gas bottle A, which is installed in the SEA of the MSPR, is regulated with $0.13 \mathrm{MPa}$ abs and supplied to the small combustion vessel through the gas inlet line. GN2 is supplied to the GCEM from the ISS via the MSPR/CCE through the GN2 supply line. The GN2 supply line has 2 branches in the GCEM. One branch is employed to release $0.15 \mathrm{MPa}$ abs GN2 inside the CCE during dilution procedures of oxygen concentration. The dilution procedures are to reduce hazard risk of fire inside the CCE, and executed in the initial set-up phase of the experiment. After the procedures, oxygen concentration inside the CCE is estimated to be lower than $10.5 \%$. Another branch supplies 0.2 MPa abs GN2 to the soot removal unit inside the small combustion vessel. Exhaust gas inside the small combustion vessel is wasted to the JEM Waste Gas Line through the gas exhaust line of the GCEM and the MSPR/CCE waste gas line. In the MSPR/CCE waste gas line, there is a filter assy to remove combustion products and fuel vapor from the exhaust gas. In the housing of the filter assy, molecular sieve $13 \mathrm{x}$ is installed as adsorbing materials.

\subsubsection{Power supply and control unit}

Power supply and control unit has the following functions.

1) To convert supplied power $(28 \mathrm{~V}, 16 \mathrm{~V})$ from the DCU of MSPR

2) To supply converted power to the GCEM components

3) To receive telecommands from the ground

4) To drive and control the GCEM components

5) To collect and store experimental data and status

6) To transfer experimental data and status to the ground

7) To perform safety functions and limitations

Power supply and control unit has thermostat inside, and its temperature is monitored electrically. In case the temperature exceeds the threshold of the thermostat, DCU of the MSPR immediately shut down power supply to the GCEM automatically. 


\subsubsection{Gas bottle A assy}

Gas bottle A assy contains dry air with $10 \mathrm{MPa}$ abs in the 2.21 inner volume. Air is supplied to the small combustion vessel from the gas bottle A assy which is installed in the SEA of the MSPR, through the air supply line. The gas bottle A assy and CCE is connected by flexible tube. For completion of whole test matrix of the Group Combustion, 5 bottles are necessary. When the amount of remaining air inside the bottle is short, crew will exchange the bottle in the SEA to new one.

\section{Development Status of the GCEM}

Proposal of the Group Combustion was selected as a candidate of the flight experiment by KIBO AO in the spring of 2008. After that, detail consideration of the experimental plan and conceptual design of the GCEM was performed in the definition phase. Then, the works of the development phase started in 2011, after the SDR (System Definition Review) and the Project Transition Review. PDR (Preliminary Design Review) was held on March, 2012. Following the result of the PDR, fabrication of the GCEM EM was started in the detail design phase. The EM was completed in the summer of 2012, and various testing was performed to confirm validity of the design.

The testing of EM includes the following items.

1) Function tests on the ground

2) Function tests in low gravity environment by parabolic flights

3) EMC (Electro Magnetic Compatibility) test

4) Fitting test with the $\mathrm{CCE}$

5) Crew review on the operability

6) Noise test

7) Measurement of the acceleration caused by the operation of the EM

8) Vibration test

Figure 9 shows a flame spread image taken by the parabolic flight tests. Functions of droplet generation, droplets supporting by the SiC fibers, ignition of droplet, etc. was confirmed in low gravity environment. Also, pressure and temperature variation in the small combustion vessel during flame spread process in low gravity environment were performed.

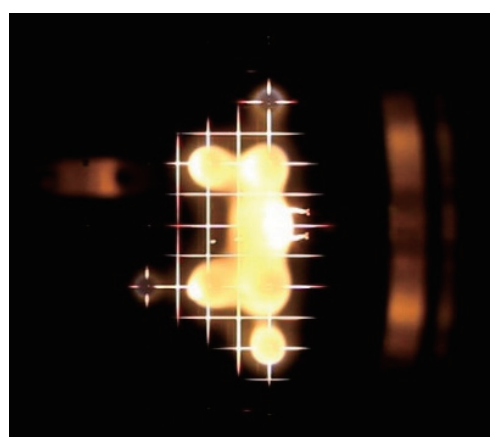

Fig. 9. Flame spread image of n-decane droplet clouds in low gravity environment by parabolic flight.

which were performed in the JAXA Tsukuba Space Center on
January 2013. The GCEM components such as GCEM main assy were installed inside the Cargo Transfer Bag (CTB) with cushion material, same as their launch configuration. After vibration test, functions of the GCEM were checked.

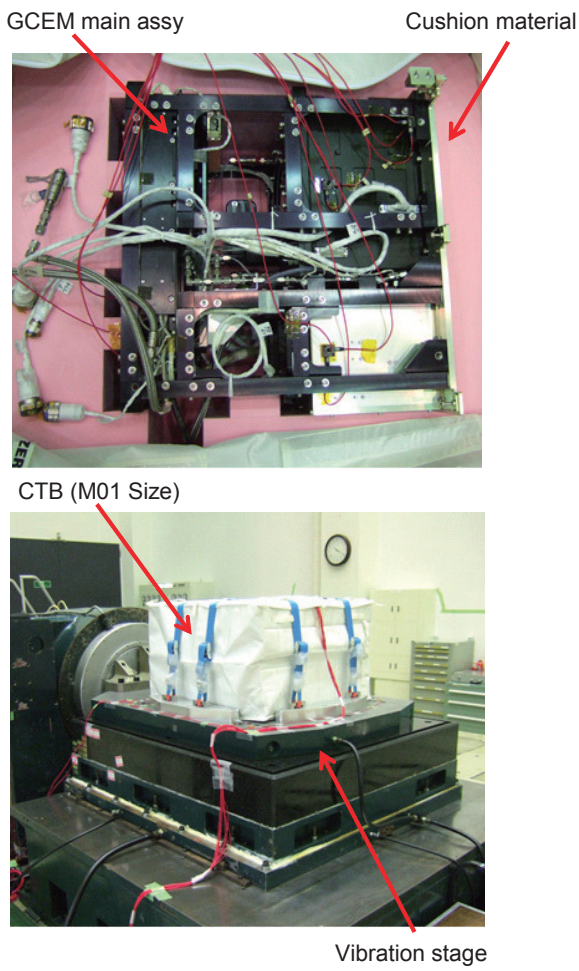

Fig. 10. Photos of the GCEM EM vibration test.

Following these tests of the EM, Critical Design Review (CDR) was performed in March 2013. As a result of CDR, fabrication of the Proto Flight Model (PFM) of the GCEM and transition to the sustainable design phase were approved with precondition of closing some action items. As of mid. April 2013, efforts for closing the action items are on-going. Fabrication of the GCEM PFM is expected to complete in this summer, and testing for verification will start immediately.

\section{Summary}

Development of the GCEM for the "Group Combustion" experiment in the KIBO has been on-going. At present, launch of the GCEM by the HTV-5 in the summer of 2014 is planned. Toward that, fabrication of the PFM will start soon.

\section{Acknowledgments}

The authors express appreciation for technical support by Mr. Seijiro Fukuyama of the AES Co., Ltd.,. Also, supports by relevant members of the Japan Space Forum (JSF) and by the Japan Manned Space Systems Corporation (JAMSS) are highly appreciated. 


\section{References}

1) Brzustowski, T. A., Sobiesiak, A. and Wojcicki, S.: Proc. Combust Inst. 18: 265-273 (1981).

2) Yoshida, S., Hara, H. and Okajima, S. JSME Trans. B (in Japanese) 55: 1241-1247 (1989).

3) Kato, S., Kobayashi, H., Mizuno, H. and Niioka, T.: JSME Int. J. B, 41: 322-330 (1998)..

4) Mikami, M., Oyagi, H., Kojima, N., Kikuchi, M., Wakashima, Y. and Yoda, S.: Combust. Flame 141: 241-252 (2005).

5) Mikami, M., Oyagi, H. Kojima, N. Wakashima, Y. Kikuchi, M. Yoda, S.: Combust. Flame, 146 (2006), pp.391-406.

6) Umemura, A.: JSME Trans. B (in Japanese) 68: (2002) 254-260.

7) Kikuchi, M., Arai, T., Yoda, S., Tsukamoto, T., Umemura, A., Uchida, M., Niioka, T.: Proc. Combust Inst., 29 (2002), pp.2611-2619.

8) Kikuchi, M., Wakashima, Y., Yoda, S., Mikami, M.: Proc. Combust Inst., 30 (2005), pp.2001-2009.

9) Kikuchi, M., Yamamoto, S., Mikami, M., Nomura, H., Moriue, O. and Umemura, A.: Proc. 49th Combustion Symposium (Japanese), 2011, pp.108-109.

10) Oyagi, H., Shigeno, H., Mikami, M., Kojima, N.: Combust. Flame 156 (2009), 763-777.

11) Shigeno, H., Oyagi, H., Mikami, M., Kojima, N.: Proc. Twenty-Fifth International Symposium on Space Technology and Science, 2006, 2006-h-24.

12) Nomura, H., Suganuma, Y., Setani, A., Takahashi, M., Mikami, M., Hara, H.: Proc. Combust. Inst., 32(2009), pp. 2163-2169. 